OPEN ACCESS

Edited by:

Nicola Smania

University of Verona, Italy

Reviewed by:

Antonino Naro,

Centro Neurolesi Bonino Pulejo

(IRCCS), Italy

Dongyu Wu

Wangjing Hospital of China Academy of Chinese Medical Sciences, China

*Correspondence:

Marousa Pavlou marousa.pavlou@kcl.ac.uk

Specialty section: This article was submitted to Neurorehabilitation,

a section of the journal

Frontiers in Neurology

Received: 17 April 2020

Accepted: 20 July 2020

Published: 26 August 2020

Citation:

Matar SJ, Sorinola IO, Newton C and

Pavlou M (2020) Transcranial

Direct-Current Stimulation May

Improve Discourse Production in Healthy Older Adults.

Front. Neurol. 11:935

doi: 10.3389/fneur.2020.00935

\section{Transcranial Direct-Current Stimulation May Improve Discourse Production in Healthy Older Adults}

\author{
Shereen J. Matar ${ }^{1}$, Isaac O. Sorinola ${ }^{2}$, Caroline Newton ${ }^{3}$ and Marousa Pavlou ${ }^{1 *}$ \\ ${ }^{1}$ Centre for Human \& Applied Physiological Sciences, Faculty of Life Sciences \& Medicine, King's College London, London, \\ United Kingdom, ${ }^{2}$ Department of Public Health Sciences, Faculty of Life Sciences \& Medicine, King's College London, \\ London, United Kingdom, ${ }^{3}$ Division of Psychology \& Language Sciences, Faculty of Brain Sciences, University College \\ London, London, United Kingdom
}

Background: The use of transcranial direct-current stimulation (tDCS) for therapeutic and neurorehabilitation purposes has become increasingly popular in recent years. Previous research has found that anodal tDCS may enhance naming ability and verbal fluency in healthy participants. However, the effect of tDCS on more functional, higher level language skills such as discourse production has yet to be understood.

Aims: The present study aimed to investigate in healthy, older adults (a) the effect of anodal tDCS on discourse production vs. sham stimulation and (b) optimal electrode placement for tDCS to target language improvement at the discourse level.

Methods: Fourteen healthy, older right-handed participants took part in this sham controlled, repeated measures pilot study. Each participant experienced three different experimental conditions; anodal tDCS on the left inferior frontal gyrus (IFG), anodal tDCS on the right IFG and sham stimulation while performing a story telling task. Significant changes in language performance before and after each condition were examined in three discourse production tasks: recount, procedural and narrative.

Results: Left and right IFG conditions showed a greater number of significant within-group improvements $(p<0.05)$ in discourse production compared to sham with 6/12 for left IFG, 4/12 for right IFG and 2/12 for sham. There were no significant differences noted between tDCS conditions. No relationship was noted between language performance and physical activity, age, or gender.

Conclusions: This study suggests that anodal tDCS may significantly improve discourse production in healthy, older adults. In line with previous tDCS language studies, the left IFG is highlighted as an optimal stimulation site for the modulation of language in healthy speakers. The findings support further exploration of tDCS as a rehabilitative tool for higher-level language skills in persons with aphasia.

Keywords: language, tDCS, neurorehabilitation, discourse, aphasia 


\section{INTRODUCTION}

The use of transcranial direct-current stimulation (tDCS) for therapeutic and neurorehabilitation purposes has become increasingly popular in recent years. Transcranial direct-current stimulation is a safe non-invasive brain stimulation (NIBS) method that can modify spontaneous cortical activity in targeted brain regions $(1,2)$. The prolonged effects of NIBS can be inhibitory or excitatory depending on polarity of current flow whereby brain excitability is often increased by anodal tDCS and decreased by cathodal tDCS (2-4). Although the exact mechanisms behind the effect of tDCS are yet to be fully understood, several studies demonstrate its effectiveness in supporting motor and cognitive function recovery, while a growing number have assessed its impact on language (5-8).

Anodal tDCS has been shown to modulate language performance in healthy speakers and in people with aphasia, including improvements in verbal fluency $(3,4,9,10)$ and naming (11-17) during word level language tasks such as picture naming. Few studies though have investigated the impact of tDCS on language performance at the discourse level $(18,19)$.

Natural verbal communication is seldom in isolated words. Discourse is a higher-level of language expanding beyond simple sentences and allows for the production of meaningful language in everyday situations $(20,21)$ including recounting information, telling stories or narratives, conversing, and giving instructions. More recently greater focus has been given to this level of language in interventions for people with aphasia. A range of interventions have shown encouraging results for improving discourse production including improved conversational ability, lexical retrieval, and syntactic structure (22-28). However, it remains unclear whether tDCS can be an effective supplementary treatment method in aphasia. Investigating the effect of tDCS on discourse production in healthy speakers can provide insight into its potential for enhancing aphasia treatment outcomes.

The effect of frontal tDCS and significance of the left inferior frontal gyrus (LIFG) in language production has been highlighted in studies assessing single word production, but discourse production is more complex and involves an interaction of linguistic and cognitive processes supported by several brain regions $(29,30)$. Although regions of the left hemisphere important for language function, including Broca's (IFG) and Wernicke's area, have been identified, the contribution of the right hemisphere to language processing is not wellestablished (31). Previous studies have found individuals with right hemispheric damage produce narratives with reduced information and organizational aspects compared to healthy speakers $(31,32)$. Additionally, imaging studies have shown regular responses in both the left and right motor cortices and bilaterally in the IFG during discourse production $(29,30)$. These findings support the involvement of the right hemisphere in discourse production. Thus, when investigating the effect of tDCS at discourse level, the right hemisphere must be considered and the LIFG cannot be assumed to be the optimal stimulation site. To date though no studies have compared the effect of left and right hemispheric tDCS on discourse speech production.
Thus, the aims of the present study were to investigate in healthy older adults the (a) effect of anodal tDCS on discourse speech production vs. sham and (b) optimal electrode placement for tDCS to target language improvement at discourse level. This study recruited healthy, older participants to be more representative of the median age of the stroke population which is 77 years old (33). Since psychological well-being, higher physical activity, and social participation levels have been linked to improved cognitive function (34-37), this study also investigated if there was a relationship between participants' language performance and these three factors. The null hypothesis assumed no significant difference between anodal tDCS and sham, whereas the alternative hypothesis assumed that all anodal tDCS conditions would result in greater language modulations and improvements in discourse production compared to sham. Changes in discourse production were expected to differ depending on electrode placement site, revealing a more optimal site for tDCS stimulation for improving discourse language skills in future studies.

\section{METHODS}

\section{Design}

This was a sham controlled, single blinded (participant) randomized repeated measures pilot tDCS study. The protocol was approved by the King's College London Research Ethics Review Board. Written informed consent was obtained from each participant. All study procedures were in accordance with the Declaration of Helsinki (38).

Each participant experienced three different experimental conditions in random order: anodal tDCS on LIFG, anodal tDCS on right IFG (RIFG), and sham. To control for order and learning effects, conditions were tested in a counterbalanced order across subjects using a Latin Square design. Participants attended four individual sessions each lasting $\sim 60 \mathrm{~min}$. Sessions were arranged 2 -weeks apart in order to decrease both the impact of carryover effects from the previous sessions and statistical bias $(39,40)$. The final 2-week follow-up assessment was performed during the fourth session.

\section{Participants}

Fourteen healthy, community-dwelling, independently mobile, older participants ( 9 females and 5 males: age range $=65-79, M$ $=73$ years) were recruited between September and November 2018. Participant inclusion criteria were healthy, older adults who were $\geq 65$ years old in order to match the current median age of persons with stroke in the UK (33), native Englishspeaking, right-handed, normal aided, or unaided visual acuity, and at least a secondary school education level. Exclusion criteria were history of neurological disease or cognitive impairment, seizures, implanted metal, or any other tDCS contraindications (2). All potential participants completed a pre-tDCS screening questionnaire to confirm study eligibility. Collected information included past medical history [i.e., any neurological, psychiatric, and/or cognitive diagnoses or symptoms; $(2,9,41,42)]$ and hand use preference in activities such as writing and eating to 
determine handedness (adapted from the Edinburgh Handedness Inventory) (43).

\section{Intervention and Blinding tDCS Stimulation}

This study followed previous protocols used in tDCS language studies $(3,15,18,44)$ as replication is encouraged to support the development of common experimental guidelines for behaviors, including language production, and allow for more efficient comparisons between tDCS studies (2). Three stimulation conditions were tested: anode over the LIFG, anode over the RIFG and sham. In line with safe guidelines (2), anodal stimulation was produced through a battery operated constant current stimulator (DC-Stimulator Plus, NeuroConn, Ilmenau, Germany). A pair of saline soaked sponge $(5 \times 7 \mathrm{~cm})$ electrodes were used to deliver the stimulation to the target area. As noted in previous studies which stimulated the IFG $(15,45,46)$, the 10-10 EEG system (47) was used as a guide for electrode placement and the stimulation electrode was placed over FC5 for LIFG and FC6 for RIFG stimulation. The cathode electrode for each condition was placed on the contralateral supraorbital ridge [Figure 1, adapted from (48)]. A constant current of $2 \mathrm{~mA}$ was applied for $20 \mathrm{~min}$ at the beginning of each session. This intensity level and duration has been found to be effective in modulating language production in healthy speakers (3, 44). During sham condition the procedure and electrode placement was identical except that tDCS was switched off after $30 \mathrm{~s}$. A ramping period of $30 \mathrm{~s}$ was applied at the beginning and end for every condition. The "study mode" option on the tDCS stimulator blinded participants to the tDCS condition $(3,10,14)$.

\section{Outcome Measures Language Tasks}

Participants attended four sessions in total: three for tDCS application and one final fourth session for 2-week follow-up testing. Sessions one to three each began with baseline language testing and ended with post-treatment testing immediately after tDCS application. Pre-post-treatment testing included production of three different discourse language tasks:

1. Recount (i.e., describing a previous holiday)

2. Procedural (i.e., describing the steps of how to make scrambled eggs) (49)

3. Narrative [i.e., retelling the Cinderella story (50)].

The same tasks were used for all participants and conditions. Participants were only provided with simple instructions for all discourse language tasks (i.e., "tell me about a past holiday"). All pre post-testing discourse language samples were recorded using a small audio recorder (TF-85, Homder, China) and orthographically transcribed for analysis.

Each tDCS application was performed with a concurrent story-telling task. Participants were presented with the wordless picture book, Frog, Where Are You (51), and asked to verbally produce a story narrative related to the pictures (29). Before beginning, participants were asked to "imagine they are telling the story to a child, providing as much detail as possible" (29).

\section{Discourse Production Measures}

Language samples from each task (recount, procedural and narrative) were analyzed for quantity and level of informativeness. The Quantitative Production Analysis [QPA $(21,52)]$ protocol was used to extract the discourse sample and measure the number of discourse words. Applied measures were based on previous studies which involved similar language samples (53-57) and included word total, verb total, utterance total, and percent Correct Information Units (\%CIU). Based on the QPA coding protocol, utterances were considered sentences if they included a predicate-argument structure (58). Verb retrieval within discourse or the process of formulating a spoken verb from a concept was measured using verb total or the count of all verb productions in a sample (23). Nicholas and Brookshire's \%CIU was used to measure the level of informativeness in each sample and was calculated by dividing the number of CIUs by the total number of words in a language sample. Words which were accurate and provided relevant information to the language task were included in the CIU word count $(59,60)$.

The following questionnaires were completed by all participants to assess for correlations between language task performance with participants' psychological wellbeing, physical activity and participation levels. The Hospital Anxiety and Depression Scale (HADS) is a 14-item scale that assesses anxiety (HAD-A) and depression (HAD-D) symptoms; scores range between 0 and 21 for both anxiety and depression subscales, where a score between 8 and 11 indicates a borderline case and a score $\geq 11$ indicates anxiety or depression $(61,62)$. The EPIC Physical Activity Questionnaires (EPAQ2) is a reliable and valid self-completed questionnaire which collects information on an individual's physical activity at home, at work and recreation. Based on total activity hours in the last 12 months, the physical activity index is applied to categorize an individual's levels of physical activity into "inactive," "moderately inactive," "moderately active," and "active" (63). The Keele Assessment of Participation (KAP) is a brief questionnaire which measures an individual's level of participation in various activities including activities of daily living, work, and social activities $(64,65)$.

\section{Statistical Analysis}

Data analysis was conducted using SPSS 25 (IBM Inc.) and Prism 8 (GraphPad Software, Inc.). Kolmogorov-Smirnov tests assessed normality and non-parametric tests were utilized in cases where normality was not met. For each tDCS condition (LIFG, RIFG, and sham) a Wilcoxon signed-ranks test was performed using false detection rate adjusted $p$-values (66) to identify significant pre-post-stimulation changes in the three language tasks for each measure. The Friedman test determined pre-stimulation baseline changes between tDCS conditions. Spearman rho tests were used to identify any relationship between language performance across tDCS conditions, participants' demographic data, and questionnaire results. The significance level was $p \leq 0.05$ for all tests. 


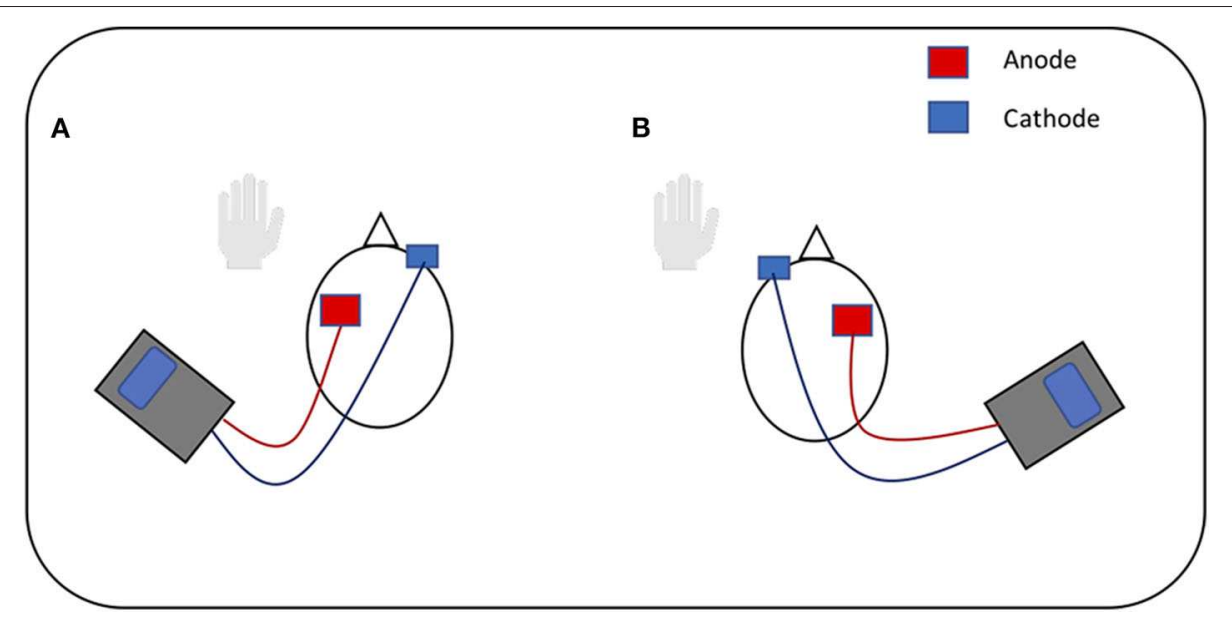

FIGURE 1 | Visual representation of tDCS. (A) Anodal stimulation of left inferior frontal gyrus: anode over the left IFG and cathode over the right supraorbital ridge. (B) Anodal stimulation of right inferior frontal gyrus: anode over the right IFG and cathode over the left supraorbital ridge. Figure adapted from Figure 1 in (48), licensed under the Creative Commons Attribution License 4.0.

TABLE 1 | Demographic data and questionnaire results for each participant.

\begin{tabular}{|c|c|c|c|c|c|}
\hline Participant & Age range & HAD-A & HAD-D & EPAQ & KAP \\
\hline & $(n=14)$ & $(n=11)$ & $(n=11)$ & $(n=11)$ & $(n=11)$ \\
\hline P01 & $76-80$ & 2 & 0 & 4 & 0 \\
\hline P02 & $71-75$ & 6 & 5 & 3 & 0 \\
\hline P03 & $65-70$ & 4 & 1 & 2 & 0 \\
\hline P04 & $76-80$ & DNC & DNC & DNC & DNC \\
\hline P05 & $71-75$ & 3 & 2 & 4 & 0 \\
\hline P06 & $76-80$ & 5 & 3 & 2 & 0 \\
\hline P07 & $71-75$ & 2 & 1 & 4 & 0 \\
\hline P08 & $65-70$ & 5 & 8 & 2 & 0 \\
\hline P09 & $76-80$ & 5 & 3 & 4 & 0 \\
\hline P10 & $76-80$ & 0 & 0 & 3 & 0 \\
\hline P11 & $71-75$ & DNC & DNC & DNC & DNC \\
\hline P12 & $65-70$ & 5 & 1 & 2 & 0 \\
\hline P13 & $65-70$ & 7 & 0 & 4 & 0 \\
\hline P14 & $71-75$ & DNC & DNC & DNC & DNC \\
\hline Mean $(S D)$ & $73(5)$ & $4(2)$ & $2(2)$ & $3(1)$ & 0 \\
\hline
\end{tabular}

DNC, did not complete; HADS, Hospital Anxiety and Depression Scale (67); EPAQ, EPIC Physical Activity Questionnaire (63); KAP, Keele Assessment of Participation (65). EPAQ physical activity index: 1 = inactive, 2 = moderately inactive, $3=$ moderately active, 4 $=$ active. KAP scoring: $0=$ no participation restrictions, $1-11=$ indication of restriction in participation in at least one activity.

\section{RESULTS}

Fourteen healthy, older adults were recruited (mean age $=73$ \pm 5; Females $=9$, Males $=5$ ). Participant demographics are presented in Table $\mathbf{1 .}$

All participants tolerated tDCS. No adverse reactions were reported or observed. All participants attended 100\% of sessions.

\section{tDCS and Language Task Performance} Pre-stimulation Assessment

No significant differences between tDCS conditions were noted in baseline pre-stimulation performances in each language task for all discourse measures.

\section{Word Total}

No significant differences between tDCS conditions were noted for word total improvements in all language tasks.

All three tDCS conditions showed a significant within-group improvement in word total for the narrative task (Figure 2; LIFG: $Z=-3.045, p=0.006$; RIFG: $Z=-3.297, p=0.005$; sham: $Z=-2.417, p=0.036$ ). In the LIFG condition $13 / 14$ participants showed a positive change (i.e., improvement) and $1 / 14$ performed worse; in RIFG condition 14/14 showed positive change; and in sham 11/14 showed positive change and 3/14 performed worse. A significant within-group improvement in recount was noted only for the LIFG condition (Figure 2; $Z$ $=-3.296, p=0.005$ ) where all 14 participants showed an improvement post-stimulation.

Improvements for the narrative task were maintained at 2week follow-up for LIFG (follow-up vs. pre-stimulation $p=$ $0.034)$ and RIFG $(p=0.028)$ conditions.

\section{Utterance Total}

No significant differences between tDCS conditions were noted for improvements in utterance total in all language tasks. For procedural, the between-group difference approached significance $(p=0.062)$ where RIFG had a greater number of utterances post-stimulation compared to LIFG and sham.

A significant pre-post-treatment change was noted with within-group improvements in utterance total for both the recount (Figure 3; $Z=-3.297, p=0.009$ ) and narrative (Figure 3; $Z=-2.657, p=0.024$ ) tasks in LIFG condition. In recount $14 / 14$ participants showed positive change and in narrative $11 / 14$ showed positive change, $1 / 14$ no change, and 

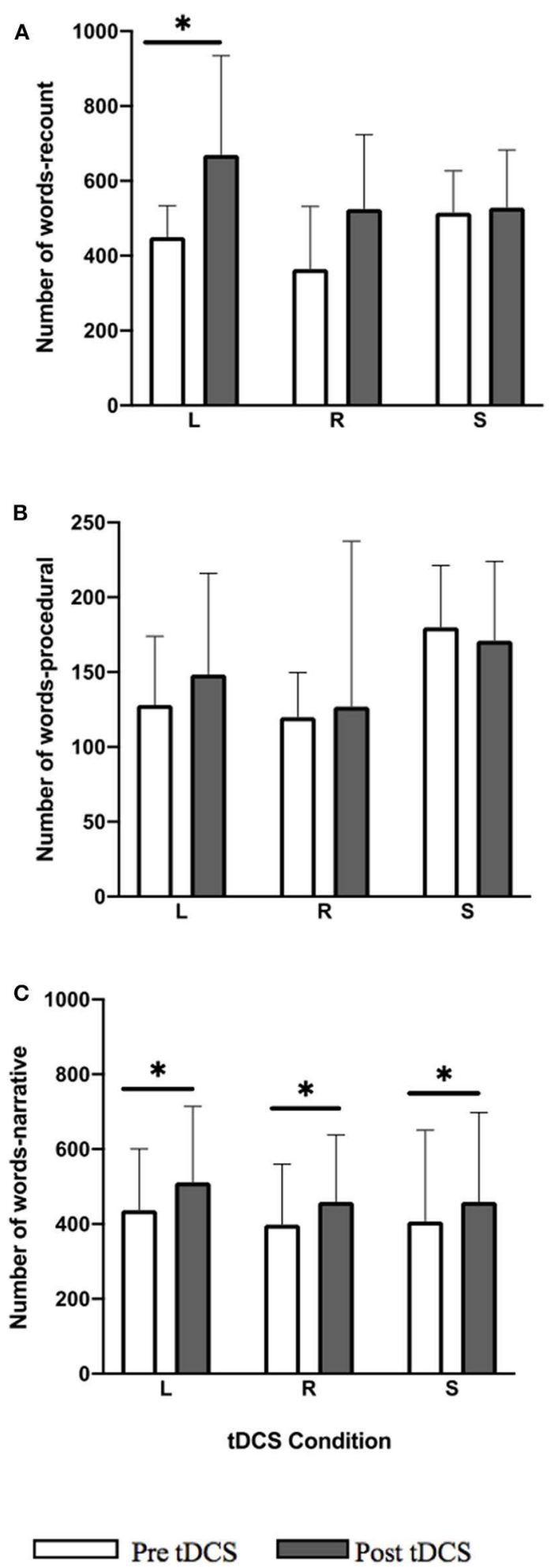

FIGURE 2 | Pre-post-stimulation group changes in word total (median values and interquartile range) in (A) recount, (B) procedural, and (C) narrative. Significant within-group differences $(p \leq 0.05)$ using false detection rate adjusted $p$-values are indicated by a $\left(^{*}\right)$. tDCS conditions are LIFG (L), RIFG $(\mathrm{R})$, and sham $(\mathrm{S})$.
2/14 performed worse. For the RIFG condition, a significant within-group improvement was noted only for the narrative task (Figure 3; $Z=-2.794, p=0.0225$ ) where $12 / 14$ participants showed positive change and $2 / 14$ performed worse. No significant improvements were noted in any of the tasks for the sham condition. Within-group improvements noted for LIFG and RIFG conditions were not maintained at follow-up.

\section{Verb Total}

No significant differences between tDCS conditions were noted for verb total improvements in all language tasks.

For the LIFG and RIFG conditions the total number of verb showed significant within-group improvements for the recount (Figure 4; LIFG: $Z=-3.297, p=0.0045$; RIFG: $Z=-2.229$, $p=0.0468$ ) and narrative (Figure 4; LIFG: $Z=-3.048, p=$ 0.006; RIFG: $Z=-3.297, p=0.0045)$ language tasks. In the recount task 14/14 participants showed positive change in LIFG condition, and 11/14 showed positive change and $3 / 14$ performed worse in RIFG condition. In the narrative task for LIFG condition 12/14 showed positive change and 2/14 performed worse and for the RIFG condition 14/14 showed positive change. For sham, post-stimulation within-group improvements in the narrative task were significant (Figure 4; $Z=-2.726, p=0.014$ ) where $11 / 14$ showed positive change, $1 / 14$ showed no change, and $2 / 14$ performed worse. Within-group improvements were not maintained at follow-up.

\section{$\% \mathrm{CIU}$}

No significant pre-post-treatment change was noted in \%CIU in all tDCS conditions for each language task: recount, procedural, and narrative (Figure 5).

\section{Questionnaire Results and Correlations}

Eleven participants completed the HADS, EPAQ, and KAP (descriptive data are presented in Table 1). Participants' $(n=11)$ scores were within normal ranges for the HAD-D and HADA except for a single participant with a HAD-A score of $8 / 21$ indicating a borderline case. EPAQ scores identified an average "moderately active" level of physical activity across participants with none in the inactive range, four in the moderately inactive range, two in the moderately active range, and five in the active range. On the KAP, all participants $(n=11)$ scored $0 / 11$, indicating no restrictions for daily participation.

Spearman rho tests revealed no significant correlations between physical activity levels, HADS scores, age, or gender and performance in language tasks across tDCS conditions.

\section{DISCUSSION}

The aim of this study was to investigate the effect of anodal tDCS on discourse production and optimal electrode placement for the enhancement of language at this level in healthy older speakers. Several discourse measures were used as this is encouraged in aphasia research in order to gain a comprehensive analysis at the level of discourse speech and identify areas of strength in discourse output $(20,68,69)$. Overall, the 

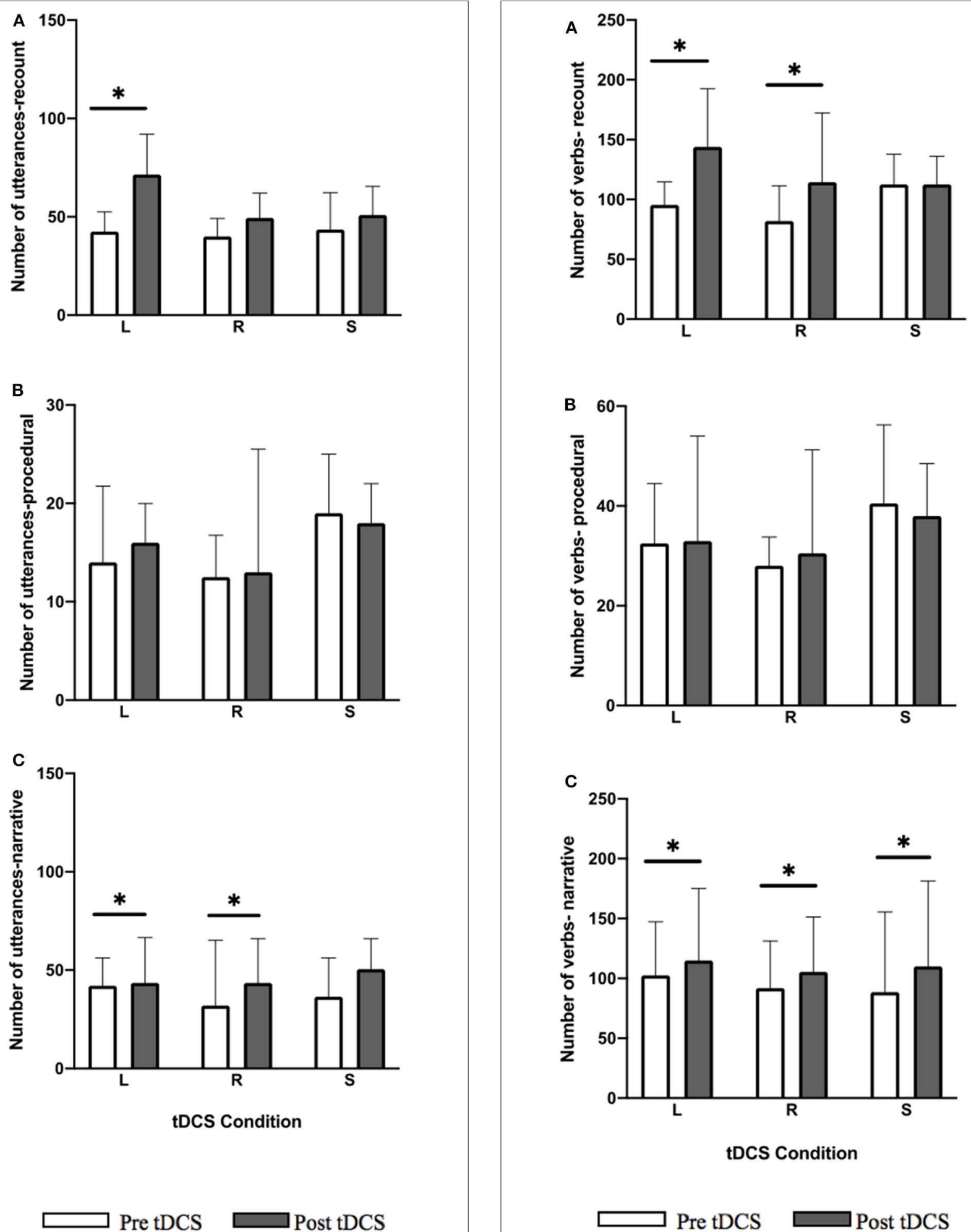

FIGURE 3 | Pre-post-stimulation group changes in utterance total (median values and interquartile range) in (A) recount, (B) procedural, and (C) narrative. Significant within-group differences $(p \leq 0.05)$ using false detection rate adjusted $p$-values are indicated by a $\left(^{*}\right)$. tDCS conditions are LIFG (L), RIFG $(R)$, and sham $(S)$. 

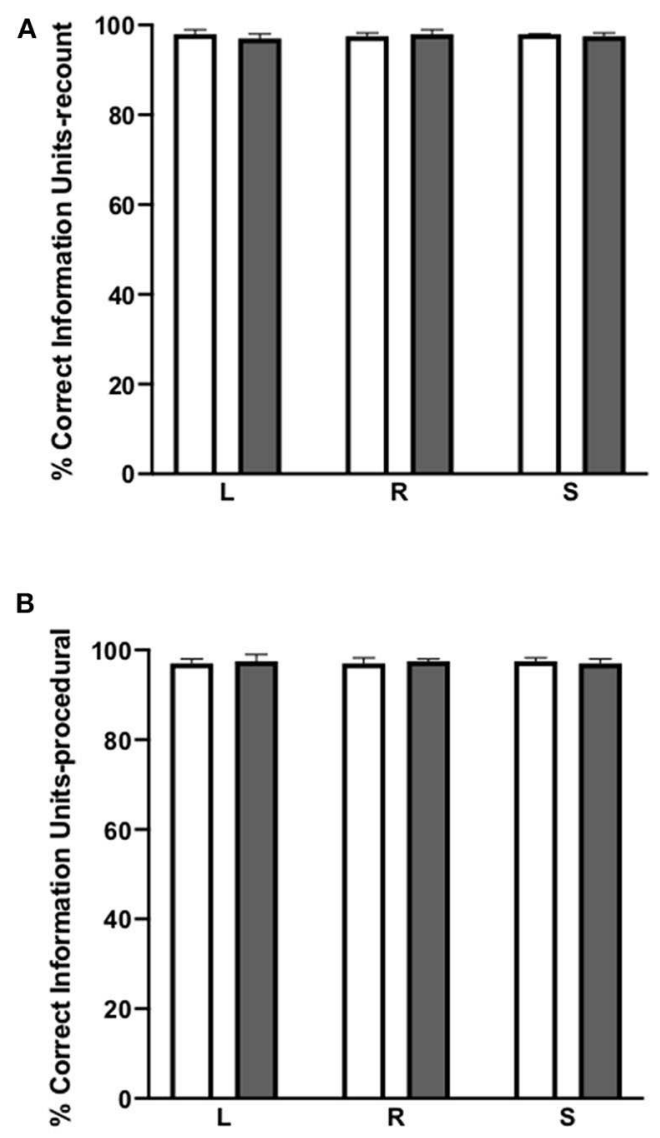

C

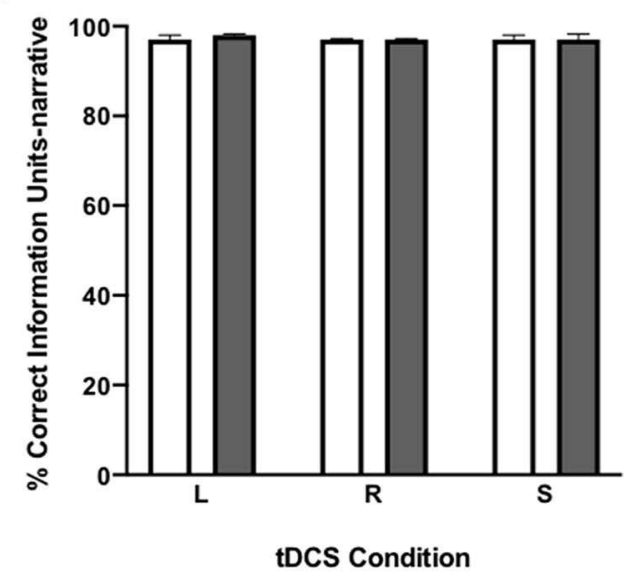

Pre tDCS

Post tDCS

FIGURE 5 | Pre-post-stimulation group changes in \%CIU (median values and interquartile range) in (A) recount, (B) procedural, and (C) narrative. tDCS conditions are LIFG (L), RIFG (R), and sham (S). results demonstrate a greater number of significant withingroup language modulations for both anodal tDCS conditions compared to sham, where the greatest improvements in language performance were noted for LIFG stimulation. The stimulation effect was not equal across discourse language tasks and there was no relationship found between language performance and psychological well-being, physical activity, age, or gender. The above main findings will be further discussed below.

Study findings demonstrate that both active anodal tDCS conditions (LIFG and RIFG) result in a greater number of significant within-group improvements in language performance than sham (6/12 for left IFG, 4/12 for right IFG, and $2 / 12$ for sham). Both anodal tDCS on LIFG and RIFG produced significant within-group improvements in recount and narrative language tasks, whereas improvements resulting from sham stimulation were limited to the narrative task. Previous work found that repeating narrative tasks may lead to some improvements in language production due to increased familiarity from using identical narrative elicitation methods (70). Therefore, although there was a 2-week washout period between sessions, the small number of significant within-group improvements in narrative noted with sham stimulation may be related to practice effects. In line with a recent review (44), these findings support the use of anodal tDCS as an effective technique for modulating language production in healthy older individuals. Previous tDCS studies in healthy speakers have demonstrated improvements within word level tasks $(5,11,13,16)$, however, we believe this is the first study indicating that the spontaneous neuron activity and cortical excitability changes caused by tDCS (71) could also enhance word retrieval and overall language performance within discourse of healthy speakers, a more complex and functional form of language production.

Most previous tDCS studies have focused on examining the impact of tDCS in younger adults. However, normal physiological aging results in a decline in language abilities, including word retrieval, due to changes in synaptic connectivity which may alter neuroplasticity mechanisms (12). The current findings are the first to indicate that tDCS may have a positive effect on higher-level language performance in healthy older adults and may be an effective method to counteract age-related changes in language abilities.

Although our findings support past imaging studies (29, 72) demonstrating an important role of the right hemisphere, including the RIFG, in discourse production, significant improvements were noted for a greater number of factors in the LIFG (6/12) compared to RIFG (4/12) condition. In some areas the RIFG condition showed similar improvements to LIFG however more improvement in the number of words and utterances in the recount task were noted only with LIFG, suggesting it as a more optimal stimulation site for improving discourse production. No significant differences were noted between tDCS conditions which may be due to the study's small sample size. Our findings are in accordance with previous research supporting the importance of the LIFG for language production of words and sentences $(73,74)$. Although the 
exact mechanisms underpinning tDCS and neuroplasticity are not clearly understood, recent functional magnetic resonance imaging (fMRI) work has sought to analyse the neural mechanisms which underlie behavioral improvements resulting from tDCS (75). Studies which combined tDCS with PMRI found that compared to sham, anodal tDCS on LIFG during different word level language tasks (i.e., naming, verb learning) decreases brain activity $(15,76,77)$ and modulates language network connectivity in healthy speakers $(45,76,77)$. Neural changes (i.e., reduced activity in LIFG) which are associated with improved behavior suggest that tDCS indirectly improves performance by promoting increased processing efficiency $(45,76)$. The number of significant improvements resulting from anodal tDCS on LIFG in this study may have resulted from neuroplastic aftereffects caused by similar neural mechanisms. However, more work is needed in this area including examining higher level language output such as discourse to better understand the neural mechanisms which allow tDCS to modulate language performance at this level (73).

The tDCS effect was not equal across the three discourse tasks. The majority of significant within-group improvements resulting from both active tDCS conditions were noted in recount language samples. Significant improvements in narrative language samples were noted in all three tDCS conditions, suggesting that tDCS has a greater impact on recounting discourse skills. No significant changes were observed for the procedural task across conditions. This discrepancy may be directly related to the language task completed during tDCS stimulation. It has been previously found that the task completed during stimulation can impact the effects of tDCS on poststimulation performance (78), where greater post-stimulation tDCS effects are observed in tasks which require similar cognitive abilities to the one completed during stimulation. In a previous study, participants who received tDCS while completing a picture naming task (nouns) had post-stimulation improvements only at the word level in the number of nouns produced, indicating that the task completed during tDCS may have promoted the production of nouns (79). In the current study the concurrent story telling task, may have supported improvements in the similar post-stimulation recount and narrative tasks which also involves the description of a sequence of events and actions that develop over time (80), but not the procedural task as no similar activities were performed during tDCS stimulation. Further studies are required to understand the specific influence of the concurrent task on post-tDCS effects.

Increasing physical activity has been found to improve cognitive function and promote neuroplasticity (35), however, the current study did not identify an association between physical activity levels and language performance. It is important to note though that the majority of participants were in the active range and none were categorized as inactive. Anxiety has been previously associated with deficits in communication (36) and depression has been linked to cognitive dysfunction (34). Since participants in this study had normal range psychological symptoms except for a single participant with a borderline caseness of anxiety, it was not possible to properly examine the association between psychological symptoms and language performance.

Brain activation in males may be lateralised to the LIFG whereas in females activation occurs bilaterally in both the left and right IFG during phonological language tasks $(81,82)$. No significant association was observed though between gender and language performance post-stimulation on RIFG and LIFG. This may be due to the more complex nature of discourse production which requires activation of both hemispheres regardless of gender. Similarly, no significant relationship was found between age and pre-post-stimulation change in language performance in this cohort of healthy older adults. Since the mean age of participants was 73 years (range $=65-79$ ), a group with a wider age range may have performed differently and have enabled us to identify an association. However, this is a pilot study and further work is required to establish if there is a relationship between discourse language performance and psychological state, physical activity, age, and gender in healthy and patient populations.

The use of sham condition to control for non-specific tDCS effects provides confidence that our results were due to anodal tDCS. There were a number of study limitations though. The small sample size reduced statistical power; electrode placement may have reduced placement accuracy as it depends on human measurement using the 10-10 EEG system as a guide; despite the 2-week washout period between sessions there may have been carryover effects; and non-blinding of the main researcher and outcome assessor to the tDCS condition introduces bias. As this study did not simultaneously investigate excitability of the stimulated frontal regions, future work incorporating imaging techniques would strengthen the hypotheses made regarding the effects of tDCS on discourse production. Additional investigations of the mechanism of tDCS in the LIFG and other language related cortical areas may establish the tDCS induced neurophysiological changes that are responsible for improving language production. Comparing the effect of tDCS on LIFG with other tDCS montages such as bi-hemispheric stimulation (i.e., bilateral IFG stimulation) still needs to be explored to confirm the optimal tDCS target for improving language production at discourse level. Finally, this study followed standard tDCS methods which typically use electrode sizes of $5 \times 7 \mathrm{~cm}$ or 5 $\times 5 \mathrm{~cm}$, however, a more focal method, HD-tDCS, which uses small ring electrodes, may provide a stronger understanding of the effects of IFG stimulation on discourse production (2).

\section{CONCLUSION}

This study demonstrates that anodal tDCS may significantly improve discourse production in healthy, older adults, and further reinforces the LIFG both as a critical region for language production and as an optimal stimulation site for the modulation of language in healthy speakers. Based on the study findings, although both LIFG and RIFG conditions produced improvements in discourse production, significant within-group improvements were greater for the LIFG condition. These findings contribute to the foundation for future clinical trials investigating the effects of tDCS on discourse production 
and support the use of tDCS as a rehabilitative tool for higher-level language skills in people with aphasia due to neurological conditions.

\section{DATA AVAILABILITY STATEMENT}

The raw data supporting the conclusions of this article are available on request to the corresponding author.

\section{ETHICS STATEMENT}

The studies involving human participants were reviewed and approved by King's College London Biomedical \& Health

\section{REFERENCES}

1. Nitsche MA, Paulus W. Excitability changes induced in the human motor cortex by weak transcranial direct current stimulation. J Physiol. (2000) 527:633-9. doi: 10.1111/j.1469-7793.2000.t01-1-00633.x

2. Thair H, Holloway AL, Newport R, Smith AD. Transcranial direct current stimulation (tDCS): a Beginner's guide for design and implementation. Front Neurosci. (2017) 11:641. doi: 10.3389/fnins.2017.00641

3. Cattaneo Z, Pisoni A, Papagno C. Transcranial direct current stimulation over Broca's region improves phonemic and semantic fluency in healthy individuals. Neuroscience. (2011) 183:6470. doi: 10.1016/j.neuroscience.2011.03.058

4. Iyer MB, Mattu U, Grafman J, Lomarev M, Sato S, Wassermann EM. Safety and cognitive effect of frontal DC brain polarization in healthy individuals. Neurology. (2005) 64:872-5. doi: 10.1212/01.WNL.0000152986. 07469.E9

5. Fiori V, Coccia M, Marinelli C V., Vecchi V, Bonifazi S, Ceravolo MG, et al. Transcranial direct current stimulation improves word retrieval in healthy and nonfluent aphasic subjects. J Cogn Neurosci. (2011) 23:230923. doi: $10.1162 /$ jocn.2010.21579

6. Bastani A, Jaberzadeh S. Does anodal transcranial direct current stimulation enhance excitability of the motor cortex and motor function in healthy individuals and subjects with stroke: a systematic review and metaanalysis. Clin Neurophysiol. (2012) 123:644-57. doi: 10.1016/j.clinph.2011. 08.029

7. Flöel A. TDCS-enhanced motor and cognitive function in neurological diseases. Neuroimage. (2014) 85:93447. doi: 10.1016/j.neuroimage.2013.05.098

8. Banissy MJ, Muggleton NG. Transcranial direct current stimulation in sports training: potential approaches. Front Hum Neurosci. (2013) 7:129. doi: 10.3389/fnhum.2013.00129

9. Pisoni A, Mattavelli G, Papagno C, Rosanova M, Casali AG, Lauro LJR. Cognitive enhancement induced by anodal tDCS drives circuit-specific cortical plasticity. Cereb Cortex. (2018) 28:1132-40. doi: 10.1093/cercor/bhx021

10. Vines BW, Norton AC, Schlaug G. Non-invasive brain stimulation enhances the effects of melodic intonation therapy. Front Psychol. (2011) 2:230. doi: 10.3389/fpsyg.2011.00230

11. Fertonani A, Rosini S, Cotelli M, Rossini PM, Miniussi C. Naming facilitation induced by transcranial direct current stimulation. Behav Brain Res. (2010) 208:311-8. doi: 10.1016/j.bbr.2009.10.030

12. Fertonani A, Brambilla M, Cotelli M, Miniussi C. The timing of cognitive plasticity in physiological aging : a tDCS study of naming. Front Aging Neurosci. (2014) 6: 131. doi: 10.3389/fnagi.2014.00131

13. Sparing R, Dafotakis M, Meister IG, Thirugnanasambandam N, Fink GR. Enhancing language performance with non-invasive brain stimulation-A transcranial direct current stimulation study in healthy humans. Neuropsychologia. (2008) 46:2618. doi: 10.1016/j.neuropsychologia.2007.07.009
Sciences, Dentistry, Medicine and Natural \& Mathematical Sciences Research Ethics Review Board. The patients/participants provided their written informed consent to participate in this study.

\section{AUTHOR CONTRIBUTIONS}

SM, IS, and MP: study concept. SM, IS, CN, and MP: development of methodology, manuscript draft revisions, and editing. SM: data collection and original draft preparation. SM and MP: data analysis. MP: supervision. All authors read and approved the final manuscript.

14. Pisoni A, Papagno C, Cattaneo Z. Neural correlates of the semantic interference effect : new evidence from transcranial direct current stimulation. Neuroscience. (2012) 223:56-67. doi: 10.1016/j.neuroscience.2012.07.046

15. Holland R, Leff AP, Josephs O, Galea JM, Desikan M, Price CJ, et al. Speech facilitation by left inferior frontal cortex stimulation. Curr Biol. (2011) 21:1403-7. doi: 10.1016/j.cub.2011.07.021

16. Ross LA, McCoy D, Wolk DA, Coslett HB, Olson IR. Improved proper name recall by electrical stimulation of the anterior temporal lobes. Neuropsychologia. (2010) 48:36714. doi: 10.1016/j.neuropsychologia.2010.07.024

17. Baker J, Rorden C, Fridriksson J. Using transcranial direct current stimulation (tDCS) to treat stroke patients with aphasia. Stroke. (2010) 41:122936. doi: 10.1161/STROKEAHA.109.576785

18. Campana S, Caltagirone C, Marangolo P. Combining voxel-based lesionsymptom mapping (VLSM) with a-tdcs language treatment: predicting outcome of recovery in nonfluent chronic aphasia. Brain Stimul. (2015) 8:769-76. doi: 10.1016/j.brs.2015.01.413

19. Galletta EE, Vogel-Eyny A. Translational treatment of aphasia combining neuromodulation and behavioral intervention for lexical retrieval: implications from a single case study. Front Hum Neurosci. (2015) 9:447. doi: 10.3389/fnhum.2015.00447

20. Armstrong E. Aphasic discourse analysis: the story so far. Aphasiology. (2000) 14:875-92. doi: 10.1080/02687030050127685

21. Saffran EM, Berndt RS, Schwartz MF. The quantitative analysis of agrammatic production: procedure and data. Brain Lang. (1989) 37:44079. doi: 10.1016/0093-934X(89)90030-8

22. Best W, Maxim J, Heilemann C, Beckley F, Johnson F, Edwards SI, et al. Conversation therapy with people with aphasia and conversation partners using video feedback: a group and case series investigation of changes in interaction. Front Hum Neurosci. (2016) 10:562. doi: $10.3389 /$ fnhum.2016.00562

23. Newton C, Kirby P, Bruce C. Getting into shape: the effect of Shape Coding on the spoken language production of two men with chronic aphasia. Aphasiology. (2017) 31:1459-81. doi: 10.1080/02687038.2017.1306639

24. Furnas DW, Edmonds LA. The effect of computerised verb network strengthening treatment on lexical retrieval in aphasia. Aphasiology. (2014) 28:401-20. doi: 10.1080/02687038.2013.869304

25. Edmonds L a., Babb M. Effect of verb network strengthening treatment in moderate-to-severe aphasia. Am J Speech-Language Pathol. (2011) 20:13145. doi: 10.1044/1058-0360(2011/10-0036)

26. Silkes JP, Fergadiotis G, Pompon RH, Kendall DL. Effects of phonomotor treatment on discourse production. Aphasiology. (2019) 33:125-39. doi: 10.1080/02687038.2018.1512080

27. McCall D, Virata T, Linebarger MC, Berndt RS. Integrating technology and targeted treatment to improve narrative production in aphasia: a case study. Aphasiology. (2009) 23:438-61. doi: 10.1080/02687030701818000

28. Fink R, Bartlett M, Lowery J, Linebarger M, Schwartz M. Aphasic speech with and without SentenceShaper $\AA$ : two methods for assessing informativeness. Aphasiology. (2008) 22:679-90. doi: 10.1080/02687030701800792 
29. Troiani V, Fernandez-Seara MA, Wang Z, Detre JA, Ash S, Grossman M. Narrative speech production: an fMRI study using continuous arterial spin labeling. Neuroimage [Internet]. (2008) 40:932-9. doi: 10.1016/j.neuroimage.2007.12.002

30. Silbert LJ, Honey CJ, Simony E, Poeppel D, Hasson U. Coupled neural systems underlie the production and comprehension of naturalistic narrative speech. Proc Natl Acad Sci. (2014) 111:E4687-96. doi: 10.1073/pnas.1323812111

31. Marini A, Carlomagno S, Caltagirone C, Nocentini U. The role played by the right hemisphere in the organization of complex textual structures. Brain Lang. (2005) 93:46-54. doi: 10.1016/j.bandl.2004.08.002

32. Marini A. Characteristics of narrative discourse processing after damage to the right hemisphere characteristics of narrative discourse processing after damage to the right hemisphere. Semin Speech Lang. (2012) 33:6878. doi: 10.1055/s-0031-1301164

33. National Institute for Health and Clinical Excellence (NICE). Strok Stroke and Transient Ischaemic Attack in Over 16s: Diagnosis and Initial Management Management. Nice Guideline. (2019). Available online at: http://www.nice. org.uk/guidance/CG68 (accessed February 13, 2020).

34. Lam RW, Kennedy SH, Mcintyre RS, Khullar A. Cognitive dysfunction in major depressive disorder: effects on psychosocial functioning and implications for treatment. Can J Psychiatry. (2014). 59:649-54. doi: 10.1177/070674371405901206

35. Erickson KI, Gildengers AG, Butters MA. Physical activity and brain plasticity in late adulthood. Dialogues Clin Neurosci. (2013) 15:99-108. Available online at: https://www.dialogues-cns.org/contents-15-1/dialoguesclinneurosci-1599/

36. Almeida AAF De, Behlau M, Leite JR. Correlation between anxiety and communicative performance. Rev Soc Bras Fonoaudiol. (2011) 16:3849. doi: 10.1590/S1516-80342011000400004

37. Glei DA, Landau DA, Goldman N, Chuang YL, Rodríguez G, Weinstein M. Participating in social activities helps preserve cognitive function: an analysis of a longitudinal, population-based study of the elderly. Int J Epidemiol. (2005) 34:864-71. doi: 10.1093/ije/dyi049

38. World Medical Association. World Medical Association Declaration of Helsinki: ehical principles for medical research involving human subjects. JAMA. (2013) 310:2191-4. doi: 10.1001/jama.2013.281053

39. Flöel A, Rösser N, Michka O, Knecht S, Breitenstein C. Noninvasive brain stimulation improves language learning. J Cogn Neurosci. (2008) 20:141522. doi: 10.1162/jocn.2008.20098

40. Marangolo P, Fiori V, Calpagnano MA, Campana S, Razzano C, Caltagirone C, et al. tDCS over the left inferior frontal cortex improves speech production in aphasia. Front Hum Neurosci. (2013) 7:539. doi: 10.3389/fnhum.2013.00539

41. Liston MB, Bamiou DE, Martin F, Hopper A, Koohi N, Luxon L, et al. Peripheral vestibular dysfunction is prevalent in older adults experiencing multiple non-syncopal falls versus age-matched non-fallers: a pilot study. Age Ageing. (2014) 43:38-43. doi: 10.1093/ageing/aft129

42. Chew T, Ho KA, Loo CK. Inter- and intra-individual variability in response to transcranial direct current stimulation (tDCS) at varying current intensities. Brain Stimul. (2015) 8:1130-7. doi: 10.1016/j.brs.2015.07.031

43. Oldfield RC. The assessment and analysis of handedness: the Edinburgh inventory. Neuropsychologia. (1971) 9:97113. doi: 10.1016/0028-3932(71)90067-4

44. Klaus J, Schutter DJLG. Brain and cognition non-invasive brain stimulation to investigate language production in healthy speakers : a meta-analysis. Brain Cogn. (2018) 123:10-22. doi: 10.1016/j.bandc.2018.02.007

45. Holland R, Leff AP, Penny WD, Rothwell JC, Crinion J. Modulation of frontal effective connectivity during speech. Neuroimage. (2016) 140:126-33. doi: 10.1016/j.neuroimage.2016. 01.037

46. Niu X, Li J, Browne GJ, Li D, Cao Q, Liu X, et al. Transcranial stimulation over right inferior frontal gyrus increases the weight given to private information during sequential decision-making. Soc Cogn Affect Neurosci. (2019) 14:5971. doi: $10.1093 /$ scan/nsy106

47. Fitzgerald PB, Maller JJ, Hoy KE, Thomson R, Daskalakis ZJ. Exploring the optimal site for the localization of dorsolateral prefrontal cortex in brain stimulation experiments. Brain Stimul. (2009) 2:234-7. doi: 10.1016/j.brs.2009.03.002
48. Fleming MK, Theologis T, Buckingham R, Johansen-Berg H. Transcranial direct current stimulation for promoting motor function in cerebral palsy: a review. J Neuroeng Rehabil. (2018) 15:121. doi: 10.1186/s12984-018-0476-6

49. Whitworth A, Leitão S, Cartwright J, Webster J, Hankey GJ, Zach J, et al. NARNIA: a new twist to an old tale. A pilot RCT to evaluate a multilevel approach to improving discourse in aphasia. Aphasiology. (2015) 29:134582. doi: 10.1080/02687038.2015.1081143

50. Whitworth A, Claessen M, Leitão S, Webster J. Beyond narrative: Is there an implicit structure to the way in which adults organise their discourse? Clin Linguist Phonetics. (2015) 29:455-81. doi: 10.3109/02699206.2015.10 20450

51. Mayer M. Frog, Where Are You? New York, NY: Penguin Books (1969).

52. Rochon E, Saffran EM, Berndt RS, Schwartz MF. Quantitative analysis of aphasic sentence production: further development and new data. Brain Lang. (2000) 72:193-218. doi: 10.1006/brln.1999.2285

53. Altman LJP, Hazamy AA, Carvajal PJ, Benjamin M, Rosenbek J c., Crosson B. Delayed stimulus-specific improvements in discourse following anomia treatment using an intentional gesture. J Speech Hear Res. (2014) 57:43954. doi: 10.1044/1092-4388(2013/12-0224)

54. Goral M, Kempler D. Training verb production in communicative context: evidence from a person with chronic non-fluent aphasia. Aphasiology. (2009) 23:1383-97. doi: 10.1080/02687030802235203

55. Knoph MIN, Simonsen HG, Lind M. Cross-linguistic transfer effects of verbproduction therapy in two cases of multilingual aphasia. Aphasiology. (2017) 31:1482-509. doi: 10.1080/02687038.2017.1358447

56. Lee J, Man G. Language recovery in aphasia following implicit structural priming training: a case study. Aphasiology. (2017) 31:1441-58. doi: 10.1080/02687038.2017.1306638

57. Rider JD, Wright HH, Marshall RC, Page JL. Using semantic feature analysis to improve contextual discourse in adults with aphasia. Am J Speech Lang Pathol. (2008) 17:161-72. doi: 10.1044/1058-0360(2008/016)

58. Maher LM, Kendall D, Swearengin JA, Rodriguez A, Leon SA, Pingel $\mathrm{K}$, et al. A pilot study of use-dependent learning in the context of constraint-induced language therapy. J Int Neuropsychol Soc. (2006) 12:84352. doi: 10.1017/S1355617706061029

59. Nicholas LE, Brookshire RH. A system for quantifying the informativeness and efficiency of the connected speech of adults with aphasia. J Speech Hear Res. (1993) 36:338-50. doi: 10.1044/jshr.3602.338

60. Duncan ES, Small SL. Imitation-based aphasia therapy increases narrative content: a case series. Clin Rehabil. (2017) 31:15007. doi: $10.1177 / 0269215517703765$

61. Turon H, Carey M, Boyes A, Hobden B, Dilworth S, Sanson-Fisher R. Agreement between a single-item measure of anxiety and depression and the Hospital Anxiety and Depression Scale: a cross-sectional study. PLoS ONE. (2019) 14:e0210111. doi: 10.1371/journal.pone.02 10111

62. Lin C, Pakpour AH. Using Hospital Anxiety and Depression Scale (HADS) on patients with epilepsy : confirmatory factor analysis and Rasch models. Seizure Eur J Epilepsy. (2017) 45:42-6. doi: 10.1016/j.seizure.2016.11.019

63. Wareham NJ, Jakes RW, Rennie KL, Mitchell J, Hennings S, Day NE. Validity and repeatability of the EPIC-norfolk physical activity questionnaire. Int $J$ Epidemiol. (2002) 31:168-74. doi: 10.1093/ije/31.1.168

64. Hermsen LAH, Terwee CB, Leone SS, Zwaard B Van Der, Smalbrugge M, Dekker J, et al. Social participation in older adults with joint pain and comorbidity; testing the measurement properties of the Dutch Keele Assessment of Participation. BMJ Open. (2013) 3:e003181. doi: 10.1136/bmjopen-2013-003181

65. Wilkie R, Peat G, Thomas E, Hooper H, Croft PR. The Keele Assessment of Participation : a new instrument to measure participation restriction in population studies. Combined Qualitative and Quantitative Examination of its psychometric properties. Qual Life Res. (2005) 14:1889-99. doi: 10.1007/s11136-005-4325-2

66. Benjamini $\mathrm{Y}$, Yosef $\mathrm{H}$. Controlling the false discovery rate - a practical and powerful approach to multiple testing. J R Stat Soci Ser B. (1995). 57:289300. doi: 10.1111/j.2517-6161.1995.tb02031.x

67. Zigmond AS, Snaith RP. The hospital anxiety and depression scale. Acta Psychiatr Scand. (1983) 67:361-70. doi: 10.1111/j.1600-0447.1983.tb09716.x 
68. Altman C, Goral M, Levy ES. Integrated narrative analysis in multilingual aphasia: the relationship among narrative structure, grammaticality, and fluency. Aphasiology. (2012) 26:1029-52. doi: 10.1080/02687038.2012.686103

69. Marini A, Andreetta S, del Tin S, Carlomagno S. A multi-level approach to the analysis of narrative language in aphasia. Aphasiology. (2011) 25:137292. doi: 10.1080/02687038.2011.584690

70. Linebarger M, McCall D, Virata T, Berndt RS. Widening the temporal window: processing support in the treatment of aphasic language production. Brain Lang. (2007) 100:53-68. doi: 10.1016/j.bandl.2006.09.001

71. Stagg CJ, Antal A, Nitsche MA. Physiology of transcranial direct current stimulation. J ECT. (2018) 34:144-52. doi: 10.1097/YCT.0000000000000510

72. Braun a R, Guillemin a, Hosey L, Varga M. The neural organization of discourse: an $\mathrm{H} 2$ 15O-PET study of narrative production in English and American sign language. Brain. (2001) 124(Pt 10):2028-44. doi: 10.1093/brain/124.10.2028

73. Crosson B, Rodriguez AD, Copland D, Fridriksson J, Krishnamurthy LC, Meinzer M, et al. Neuroplasticity and aphasia treatments : new approaches for an old problem. J Neurol Neurosurg Psychiatry. (2019) 90:114755. doi: 10.1136/jnnp-2018-319649

74. Müller R, Robert D, Behen ME, Muzik O, Mangner TJ, Chugani HT. Receptive and expressive language activations for sentences : a PET study. Neuroreport. (1997). 8:3767-70. doi: 10.1097/00001756-199712010-00022

75. Meinzer M, Lindenberg R, Darkow R, Ulm L, Copland D, Flöel A. Transcranial direct current stimulation and simultaneous functional magnetic resonance imaging. J Vis Exp. (2014) 86:51730. doi: 10.3791/51730

76. Fiori V, Kunz L, Kuhnke P, Marangolo P, Hartwigsen G. Transcranial direct current stimulation (tDCS) facilitates verb learning by altering effective connectivity in the healthy brain. Neuroimage. (2018) 181:5509. doi: 10.1016/j.neuroimage.2018.07.040

77. Meinzer M, Antonenko D, Lindenberg R, Hetzer S, Ulm L, Avirame $\mathrm{K}$, et al. Electrical brain stimulation improves cognitive performance by modulating functional connectivity and task-specific activation. J Neurosci. (2012) 32:1859-66. doi: 10.1523/JNEUROSCI.4812-11.2012

78. Gill J, Shah-basak PP, Hamilton R. Brain stimulation it ' $s$ the thought that counts : examining the task-dependent effects of transcranial direct current stimulation on executive function. Brain Stimul. (2015) 8:2539. doi: 10.1016/j.brs.2014.10.018

79. Norise C, Sacchetti D, Hamilton R. Transcranial direct current stimulation in post-stroke chronic aphasia: the impact of baseline severity and task specificity in a pilot sample. Front Hum Neurosci. (2017) 11:260. doi: 10.3389/fnhum.2017.00260

80. Mar RA. The neuropsychology of narrative: story comprehension, story production and their interrelation. Neuropsychologia. (2004) 1414-34. doi: 10.1016/j.neuropsychologia.2003.12.016

81. Shaywltz BA, Shaywltz SE, Pugh KR, Constable RT, Skudlarskl P, Fulbrlght RK, et al. Sex differences in the functional organization of the brain for language. Nature. (1995) 373:607-9. doi: 10.1038/373607a0

82. Clements AM, Rimrodt SL, Abel JR, Blankner JG, Mostofsky SH, Pekar JJ, et al. Sex differences in cerebral laterality of language and visuospatial processing. Brain Lang. (2006) 98:150-8. doi: 10.1016/j.bandl.2006.04.007

Conflict of Interest: The authors declare that the research was conducted in the absence of any commercial or financial relationships that could be construed as a potential conflict of interest.

Copyright (C) 2020 Matar, Sorinola, Newton and Pavlou. This is an open-access article distributed under the terms of the Creative Commons Attribution License (CC BY). The use, distribution or reproduction in other forums is permitted, provided the original author(s) and the copyright owner(s) are credited and that the original publication in this journal is cited, in accordance with accepted academic practice. No use, distribution or reproduction is permitted which does not comply with these terms. 